\title{
Processamento de aço API 5L X70 por laminação a morno e nitretação a plasma
}

\author{
Processing of API 5L X70 steel by warm \\ rolling and plasma nitriding
}

\author{
André Sales Aguiar Furtado ${ }^{1}$, João Rodrigues de Barros Neto ${ }^{2}$, \\ Petteson Linniker Carvalho Serra ${ }^{1}$, Rômulo Ribeiro Magalhães de Sousa ${ }^{1}$
}

\footnotetext{
${ }^{1}$ Universidade Federal do Piauí, Laboratório de Plasma - PPGCEM/UFPI, Teresina, PI, Brasil.

${ }^{2}$ Universidade Federal do Piauí, Departamento de Engenharia de Materiais - UFPI, Teresina, PI, Brasil. e-mail: salesandre7@gmail.com, plinniker@gmail.com, romulorms@gmail.com, joaorbneto@gmail.com
}

\section{RESUMO}

A necessidade cada vez maior de transportar grandes quantidades de petróleo e gás em ambientes agressivos fez surgir uma demanda por aços de alta resistência e baixa liga (ARBL) de classificação API (American Petroleum Institute). O principal método de falha é, sem dúvida, as trincas induzidas por hidrogênio (TIH), dessa forma há uma necessidade de buscar alternativas metalúrgicas para diminuir a susceptibilidade desses materiais a esse fenômeno e algumas dessas soluções é a laminação a morno e nitretação a plasma. O presente estudo tem como objetivo avaliar a influência da laminação a morno na microestrutura e formação da camada nitretada em aço API 5L X70, para isso, amostras do aço em questão foram submetidas a laminação a morno com $49,4 \%$ de redução com temperatura inicial de $863^{\circ} \mathrm{C}$ e final de $689^{\circ} \mathrm{C}$, posteriormente, foi realizado nitretação a plasma convencional e com auxílio de gaiola catódica usando como parâmetros: $450{ }^{\circ} \mathrm{C}$, com fluxo de gás $75 \% \mathrm{H}_{2}-25 \% \mathrm{~N}_{2}$ e $25 \% \mathrm{H}_{2}-75 \% \mathrm{~N}_{2}$, pressão 2,5 Torr. Para a caracterização, foram realizados microscopia ótica, ensaio de microdureza Vickers e difração de raios-X. Os resultados obtidos mostram que a microestrutura apresentou um menor bandeamento, em relação à microestrutura do material como recebido, através de uma maior distribuição de perlita causada pela laminação a morno. A nitretação formou uma fina camada de compostos de dureza superficial de até 670,0 HV (HV 0.05) formadas por nitretos do tipo $\varepsilon\left(\mathrm{Fe}_{3} \mathrm{~N}\right)$ e $\gamma^{\prime}\left(\mathrm{Fe}_{4} \mathrm{~N}\right)$. Estas características são importantes no combate às trincas induzidas por hidrogênio, e sugerem que a combinação dessas duas técnicas pode ser promissora.

Palavras-chave: Aço API 5L X70, laminação a morno, nitretação a plasma, gaiola catódica.

\section{ABSTRACT}

The increasing need to transport large quantities of oil and gas in aggressive environments has led to a demand for high strength and low alloy steels (HSLA) of API (American Petroleum Institute) grade. No doubt, the main failure method of these steels is hydrogen-induced cracking (HIC), so there is a need to look for metallurgical alternatives to reduce the susceptibility of these materials to this phenomenon and some of these solutions is the warm rolling and plasma nitriding. The present study aims on evaluating the influence of the warm rolling on the microstructure and formation of the nitrided layer in API 5L X70 steel. For this, samples of the steel in question were submitted to controlled warm rolling with $49.4 \%$ reduction with initial temperature $863{ }^{\circ} \mathrm{C}$ and final temperature $689{ }^{\circ} \mathrm{C}$, posteriorly, conventional plasma nitriding and using cathodic cage in parameters were performed: $450{ }^{\circ} \mathrm{C}$, with a gas flow of $75 \% \mathrm{H}_{2}-25 \% \mathrm{~N}_{2}$ and $25 \% \mathrm{H}_{2}-75 \%$ $\mathrm{N}_{2}$, pressure 2.5 Torr. For the characterization, optical microscopy, Vickers microhardness test and X-ray diffraction were performed. The obtained results show that the microstructure presented a lower banding, in relation of the material as received, through a higher distribution of perlite caused by the warm rolling. The nitriding formed a thin layer of compounds of superficial hardness of up to $670.0 \mathrm{HV}$ (HV 0.05) formed by nitrides of type $\left(\mathrm{Fe}_{3} \mathrm{~N}\right)$ and $\gamma^{\prime}\left(\mathrm{Fe}_{4} \mathrm{~N}\right)$. These characteristics are important in the fight against hydrogeninduced cracking, and suggest that the combination of these two techniques may be promising.

Keywords: API 5L X70 Steel, warm rolling, plasma nitriding, cathodic cage.

\section{INTRODUÇÃO}


Nas últimas décadas o mundo vivenciou um aumento da demanda por óleo e seus derivados, essa crescente necessidade e o fato de muitos países objetivarem a autonomia da sua matriz energética têm impulsionado a exploração desses insumos em ambientes severos (alta pressão, grande profundidade e baixa temperatura), remotos e corrosivos (presença de $\mathrm{H}_{2} \mathrm{~S}$ ). Naturalmente houve a necessidade de transportar com segurança e de forma econômica esses produtos, e uma dessas formas é através de gasodutos. O desenvolvimento tecnológico nos processos de soldagem, uso de elementos microligantes e processos termomecânicos controlados (PTMC) possibilitaram a produção de aços baixo carbono de alta resistência para serem utilizados nessas aplicações. Isso provocou uma redução do consumo especídfico de aço devido à obtenção de gasodutos para transporte de fipetróleo e gás de maior resistência com paredes de menor espessura. Atualmente os mais utilizados para as finalidades citadas são aqueles especificados pela norma API 5L [1-5].

O principal método de falha em aços de tubulações em ambientes corrosivos de óleo e gás, é sem dúvida, devido a TIH. O hidrogênio atômico produzido durante a corrosão da superfície do duto se difunde para o interior do metal e se acomoda em defeitos como inclusões, precipitados, interfaces de fases, ilhas de martensita, e contornos de grãos. Esses átomos de hidrogênio se recombinam, formando moléculas de gás hidrogênio, criando uma pressão interna no metal. Isso reduz a ductilidade, dureza e outras propriedades mecânicas, chamado de fragilização por hidrogênio (FH) [6-8].

A microestrutura dos aços usados para tubulações de transporte de petróleo e gás tem um papel importante no mecanismo de formação e propagação de trinca, uma vez que ela determinará a densidade e tipos de sítios de aprisionamento de hidrogênio. Algumas características microestruturais, como ausência de segregação, microestrutura homogênea e baixo volume e distribuição de perlita favorecem o aumento de resistência ao TIH [9-11]. De acordo com NAFISI et al. [12] e MASOUMI e ABREU [13], é possível obter um aumento da resistência ao TIH através de aplicação de laminação a morno controlada - entre a metade da temperatura de fusão e temperatura de não-recristalização $\left(\mathrm{T}_{\mathrm{NR}}\right)$ - em aços API, obtendo microestruturas com as características citadas, além de desenvolvimento de textura cristalográfica favorável.

Estudos prévios revelaram que tratamentos superficiais na superfície do aço podem prevenir ou diminuir a entrada de hidrogênio no material metálico. FORCEY et al. [14] reportou que a formação de filme de $\mathrm{Al}_{2} \mathrm{O}_{3}$ com $1 \mu \mathrm{m}$ de espessura na superfície de aço inoxidável $316 \mathrm{~L}$, pode reduzir de 3 a 4 vezes a permeação de hidrogênio. ZHANG [15] obteve um filme de óxido que reduziu o índice de fragilização por hidrogênio em aço X80. YAMABE et al. [16] analisou dois revestimentos (Alumina/Fe-Al e Alumina/Aluminio/Fe-Al) depositados sobre duto cilíndrico de aço inoxidável 304 e ambos apresentaram alta resistência a entrada de hidrogênio. MICHLER [17] realizou nitretação a plasma em aço inoxidável austenítico DIN 1.4301 e relatou que o material nitretado não apresentou trincas durante ensaio de tração sob atmosfera do hidrogênio, diferentemente do material não nitretado. O uso de nitretação a plasma para tratamento superficial de aço API 5L $\mathrm{X} 70$ foi fracamente reportado na literatura e isso motivou a realização desse trabalho.

A nitretação a plasma possui resulta na formação de uma estrutura com duas camadas, uma superficial composta por nitretos $\mathrm{MeN}_{\mathrm{x}}$ e uma camada de difusão subjacente, onde $\mathrm{N}$ se encontra como defeito intersticial/solução sólida. Em relação a fragilização por hidrogênio, a nitretação parece apropriada pois forma uma superfície que regula os fenômenos de adsorção, dissociação, absorção e difusão para que o H não possa entrar na rede, além disso, introduz $\mathrm{N}$ intersticial que bloqueia os sítios de ancoramento de $\mathrm{H}$, reduzindo a velocidade de difusão, e cria tensões internas de compressão que podem neutralizar tensões de trações operacionais [17-20].

Como desvantagens da nitretação a plasma podemos listar o efeito de borda, efeito catodo oco, temperatura não uniforme e abertura de arcos, especialmente no tratamento de peças de geometria complexa. Recentemente, um novo dispositivo denominado gaiola catódica, desenvolvido por SOUSA et al. [21] (depósito de patente $n^{\circ}$ PI 0603213-3) com geometria bem definida, baseado no processo tela ativa (active screen), nesse método o potencial catódico e consequentemente o plasma atua na tela e não na amostra, pois a mesma estará em potencial flutuante ou sujeitos a baixa tensão de polarização. Como o plasma não atua diretamente na superfície das peças a serem tratadas, os defeitos inerentes da nitretação a plasma convencional são eliminados, além disso, pode-se obter uma temperatura de tratamento uniforme [21-27].

Diante do exposto, o objetivo desse trabalho foi analisar a alteração microestrutural e superficial devido a aplicação de processo termomecânico de laminação a morno controlada e o processo termoquímico de nitretação a plasma em aço API 5L X70 a fim de observar características indicativas de que a combinação dessas duas técnicas possa ser usada como alternativa para aumento da resistência ao TIH em aços para transporte de petróleo e gás. 


\section{MATERIAIS E MÉTODOS}

O material de partida inicial utilizado foi uma chapa de aço API 5L X70 com 8,7 mm de espessura. A composição química está especificada na Tabela 1.

Tabela 1: Composição química (Espectrometria de Emissão Ótica com Plasma) do API 5L X70 (\%p).

\begin{tabular}{l|l|l|l|l|l|l}
\hline $\mathbf{C}$ & $\mathbf{S i}$ & $\mathbf{M n}$ & $\mathbf{S}$ & $\mathbf{A l}$ & $\mathbf{C u}$ & $\mathbf{C r}$ \\
\hline 0,0991 & 0,2585 & 1,6641 & 0,0059 & 0,042 & 0,0143 & 0,0214 \\
\hline $\mathbf{P}$ & $\mathbf{N i}$ & $\mathbf{M o}$ & $\mathbf{N b}$ & $\mathbf{T i}$ & $\mathbf{V}$ & Nióbio+Vanádio+Titânio \\
\hline 0,0182 & 0,0223 & 0,8164 & 0,0615 & 0,019 & 0,0496 & 0,1302 \\
\hline
\end{tabular}

Para homogeneização, dissolver inclusões e eliminar possíveis zonas de segregação, as peças iniciais foram aquecidas à $1200{ }^{\circ} \mathrm{C}$ por 1 hora em um forno tipo mufla de especificação EDG F1200 ${ }^{\circ} \mathrm{C}$. A chapa de $8,7 \mathrm{~mm}$, após o aquecimento, foi submetida a laminação a morno controlada em três passes até uma redução de 49,4 \%, em temperaturas abaixo da $\mathrm{T}_{\mathrm{NR}}-$ calculada de acordo com FARAHAT [28], como mostra a Figura 1. Esse procedimento tomou como referência a utilizada por MASOUMI [13]. Após a laminação as chapas de aço foram cortadas, para padronização dimensional e serem tratadas duas a duas no processo de nitretação, em pequenas amostras de tamanho 15 x $15 \mathrm{~mm}$, de tamanho utilizando cortadeira metalográfica. As amostras sem laminação foram nomeadas como SL, e amostras com laminação nomeadas de C1.

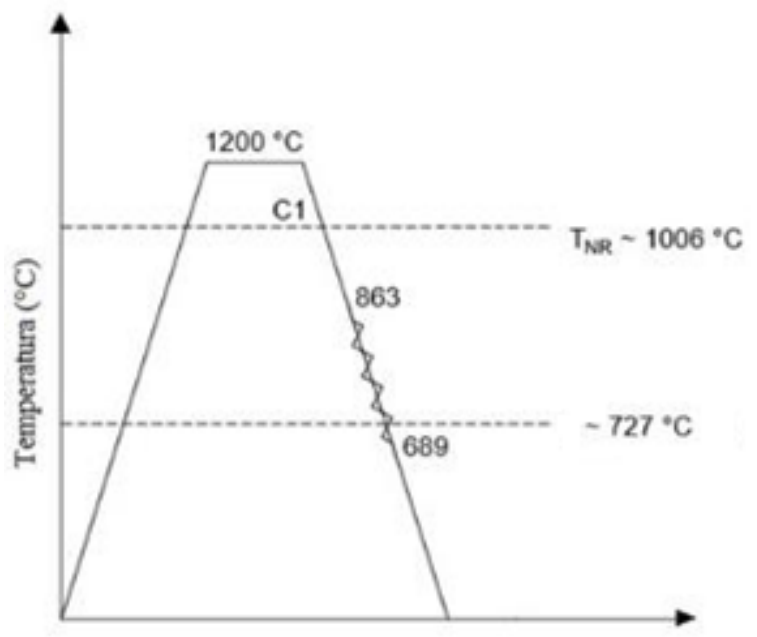

Figura 1: Esquema do processo de laminação a morno utilizado.

Para o tratamento de nitretação, as amostras foram preparadas com lixas d'água de granulometria 180, 360, 400, 600 e 1200 mesh e posteriormente polidas com pasta de diamante com granulometria $3 \mu \mathrm{m}$ até adquirir um aspecto espelhado. Os tratamentos de nitretação foram realizados em um reator da marca SDS Plasma de fonte pulsada. Duas amostras de cada condição, SL e C1 e a gaiola, foram imersas em béquer com acetona e inseridas em um equipamento de ultrassom afim de remover impurezas, posteriormente foram submetidas a tratamentos de nitretação a plasma convencional e utilizando gaiola catódica. Os parâmetros utilizados para nitretação são apresentados na Tabela 2. Embora a nitretação tenha acontecido em amostras pequenas do material em questão, ela pode ser reproduzida em dutos de tamanho padrão.

Tabela 2: Parâmetros utilizados na nitretação a plasma.

\begin{tabular}{l|l|l|l|l}
\hline \multirow{2}{*}{ PARÂMETROS } & \multicolumn{2}{|l|}{ NITRETAÇÃO A PLASMA CONVENCIONAL } & \multicolumn{2}{l}{ NITRETAÇÃO COM GAIOLA CATÓDICA } \\
\cline { 2 - 5 } & PRÉ-SPUTTERING & TRATAMENTO & PRÉ-SPUTTERING & TRATAMENTO \\
\hline Temperatura & $350{ }^{\circ} \mathrm{C}$ & $450{ }^{\circ} \mathrm{C}$ & $350{ }^{\circ} \mathrm{C}$ & $450{ }^{\circ} \mathrm{C}$ \\
\hline Pressão & $1,5 \mathrm{Torr}$ & $2,5 \mathrm{Torr}$ & $1,5 \mathrm{Torr}$ & $2,5 \mathrm{Torr}$ \\
\hline Fluxo de gás & $50 \% \mathrm{H}_{2}-50 \% \mathrm{Ar}$ & $75 \% \mathrm{H}_{2}-25 \% \mathrm{~N}_{2}$ & $50 \% \mathrm{H}_{2}-50 \% \mathrm{Ar}$ & $25 \% \mathrm{H}_{2}-75 \% \mathrm{~N}_{2}$ \\
\hline
\end{tabular}




\begin{tabular}{l|l|l|l|l}
\hline Tempo & $1 \mathrm{~h}$ & $4 \mathrm{~h}$ & $1 \mathrm{~h}$ & $4 \mathrm{~h}$ \\
\hline
\end{tabular}

A gaiola catódica utilizada nesse trabalho consiste de uma tela de aço inoxidável austenítico AISI 316 com altura $30 \mathrm{~mm}$ e diâmetro $50 \mathrm{~mm}$, a tampa da gaiola consiste em um disco também de aço inoxidável. A escolha desse tipo de material para a gaiola foi para impedir que a mesma sofra oxidação e possa ser utilizada posteriormente mas poderia ser de outra liga de aço, inclusive de aço API 5L X70. As paredes da gaiola contem furos com $8 \mathrm{~mm}$ de diâmetro com distâncias entre os centros de furos adjacentes de 9,2 mm, ela é posicionada no porta-amostras do reator e envolve as amostras de aço API que estão sobre um disco de alumina com $40 \mathrm{~mm}$ de diâmetro e $3 \mathrm{~mm}$ de espessura para que estas estejam em potencial elétrico flutuante, dessa forma o potencial elétrico irá atuar apenas na gaiola e não sobre as amostras. A Figura 2 apresenta o esquema utilizado no processo.

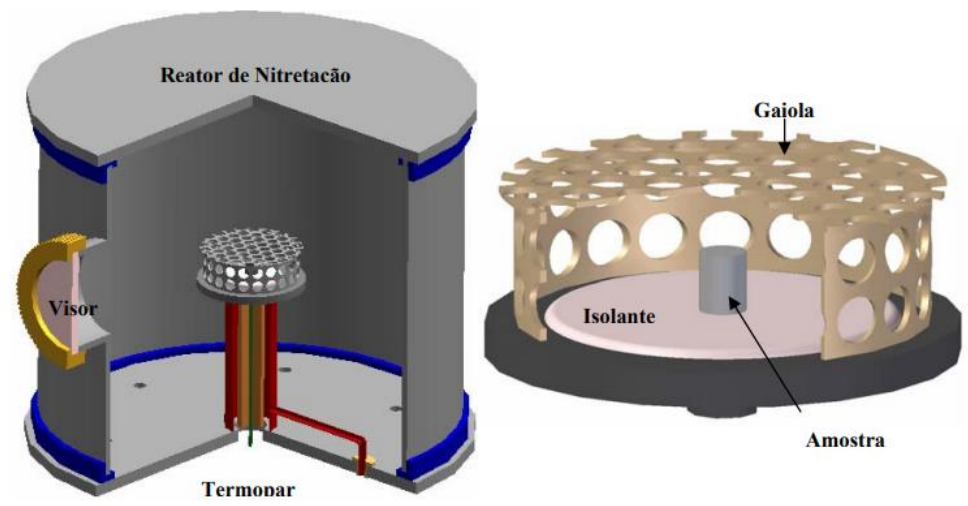

Figura 2: Vista em corte de um reator de nitretação a plasma com adaptação para gaiola catódica [29].

A identificação das amostras submetidas a nitretação seguiu a seguinte sigla: XXTNTP; (XX) - condição de laminação; (TN) - tipo de nitretação; (TP) - Temperatura de tratamento. Por exemplo, a amostra SLNC450 significa amostra sem laminação (SL) submetida a nitretação a plasma convencional na temperatura de $450{ }^{\circ} \mathrm{C}$; C1GC450 significa amostra laminada $(\mathrm{C} 1)$ submetida a nitretação a plasma com gaiola catódica na temperatura de $450{ }^{\circ} \mathrm{C}$.

Para a análise microestrutural, as amostras foram cortadas no sentido de laminação com cortadeira metalográfica e suas seções transversais foram embutidas em baquelite e passaram por procedimento padrão na metalografia que consiste em lixamento com lixas d'água SiC de granulometria 180, 360, 400, 600 e 1200 mesh e posteriormente polidas com pasta de diamante com granulometria $3 \mu \mathrm{m}$. Posteriormente, as amostras foram atacadas com reagente Nital - $2 \%$ para determinar a microestrutura e observar a camada nitretada usando um microscópio ótico BEL - modelo MTM - 1A. As medidas de microdureza na escala Vickers foram baseadas na norma ASTM E-384 e realizado na superfície (topo) de todas as amostras. Cada resultado de microdureza foi obtido através de uma média de sete identações com carga de 50 gf (HV 0,05) e tempo de aplicação de 15 segundos utilizando o microdurômetro Shimadzu modelo HMV 2000

As análises de Difração de Raios-X, para determinar as fases presentes nas camadas nitretadas e material base, foram realizadas em um difratômetro de Raios-X Philips (modelo X'PERT, radiação Co-K $(\lambda=$ $1.79026 \AA$ ) ), operando com tubo de alvo de cobalto a uma tensão de 40,0 kV e 45,0 mA de corrente, ângulo de varredura $(2 \theta)$ de $40^{\circ}$ a $100^{\circ}$ com passo angular de $0,02^{\circ}$.

\section{RESULTADOS}

A Figura 3 apresenta as micrografias do material como recebido, submetido a laminação a morno, nitretação a plasma convencional e com gaiola catódica. Analisando as imagens das Figuras 3 (a), e (b), pode-se perceber a presença de duas fases, a ferrita (fase mais clara) e perlita (mais escura). Essa microestrutura é característica desse tipo de material e também já foi observada por outros autores $[10,11,13]$.

Observando as Figuras 3 (c), (d), (e), e (f), que apresentam as micrografias das amostras nitretadas, pode-se perceber a formação de uma camada de compostos de pequena espessura (Tabela 3) em todas as amostras submetidas a nitretação, com destaque para a amostra C1NC450 que apresentou menor espessura e desvio padrão. 

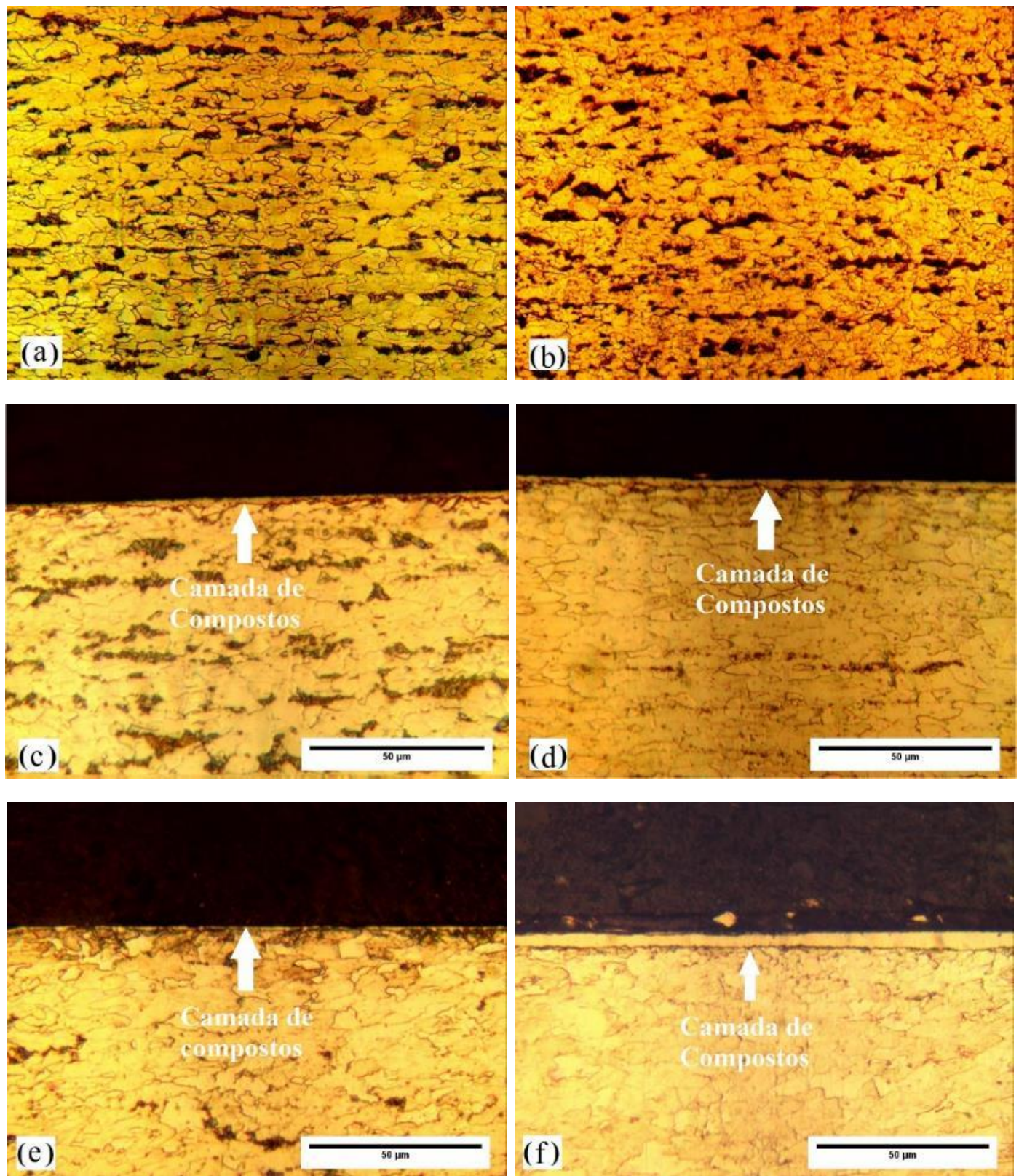

Figura 3: Micrografias das amostras SL (a), C1 (b); Aumento de 500x, SLNC450 (c), SLGC450 (d), C1NC40 (e), C1GC450 (f); aumento de 800x; Ataque com Nital 2\%.

Tabela 3: Valores de espessura de camada de compostos das amostras nitretadas.

\begin{tabular}{lll}
\hline CONDIÇÃO DE LAMINAÇÃO & NC450 & GC450 \\
\hline SL $(8,70 \mathrm{~mm})$ & $1,4 \mu \mathrm{m} \pm 0,37$ & $2,2 \mu \mathrm{m} \pm 0,34$ \\
C1 $(4,29 \mathrm{~mm})$ & $0,8 \mu \mathrm{m} \pm 0,13$ & $3,3 \mu \mathrm{m} \pm 0,31$ \\
\hline
\end{tabular}

A Figura 4 traz os resultados de ensaio de microdureza de topo do material como recebido, laminado e nitretado. Pode-se perceber uma pequena diferença entre a microdureza das amostras SL e C1 e atestar um aumento significativo da dureza superficial das amostras submetidas a nitretação a plasma convencional e com gaiola catódica, obtendo um valor máximo de 670,0 HV na amostra C1GC450, um aumento de aproximadamente $350 \%$ em relação ao material de partida $\mathrm{C} 1$. 


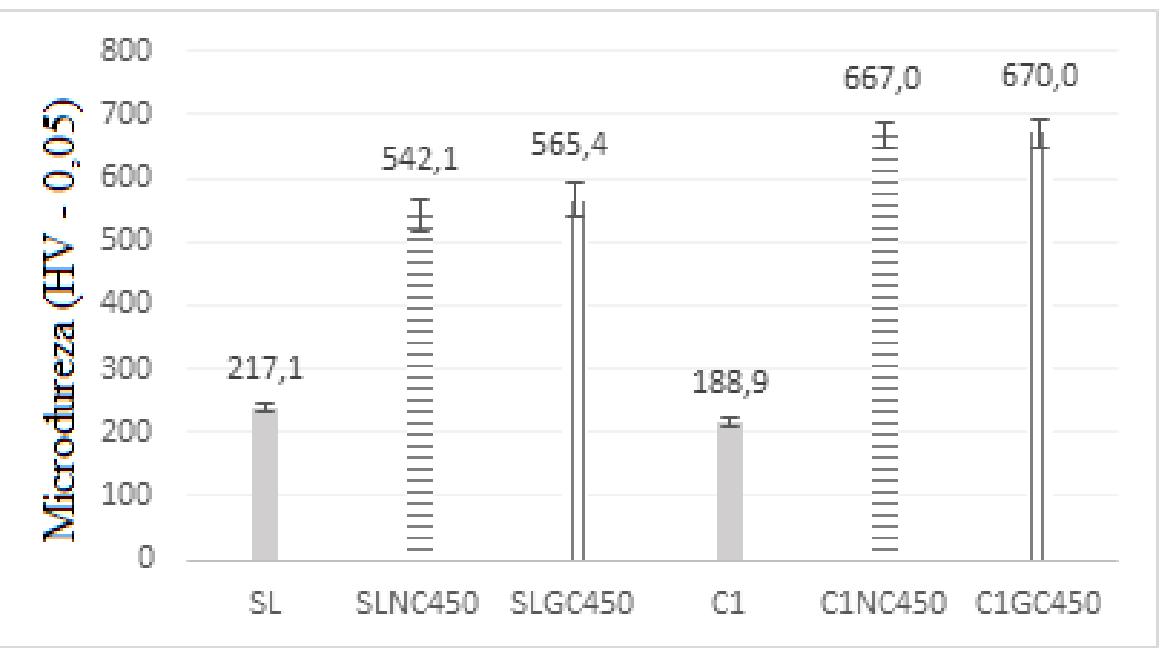

Figura 4: Resultados de microdureza de topo na escala Vickers para as amostras com e sem nitretação.

A Figura 5 apresenta os padrões de difração de raios-x das amostras analisadas, os difratogramas das amostras SL e C1 apresentam picos característicos da fase ferrita $(\alpha)$, fase de maior volume em materiais de baixo carbono. Ao observar os difratogramas do material nitretado, é possível identificar as fases $\mathrm{Fe}_{4} \mathrm{~N}\left(\gamma^{\prime}\right)$, $\mathrm{Fe}_{2-3} \mathrm{~N}(\varepsilon)$ e ferrita $(\alpha)$.
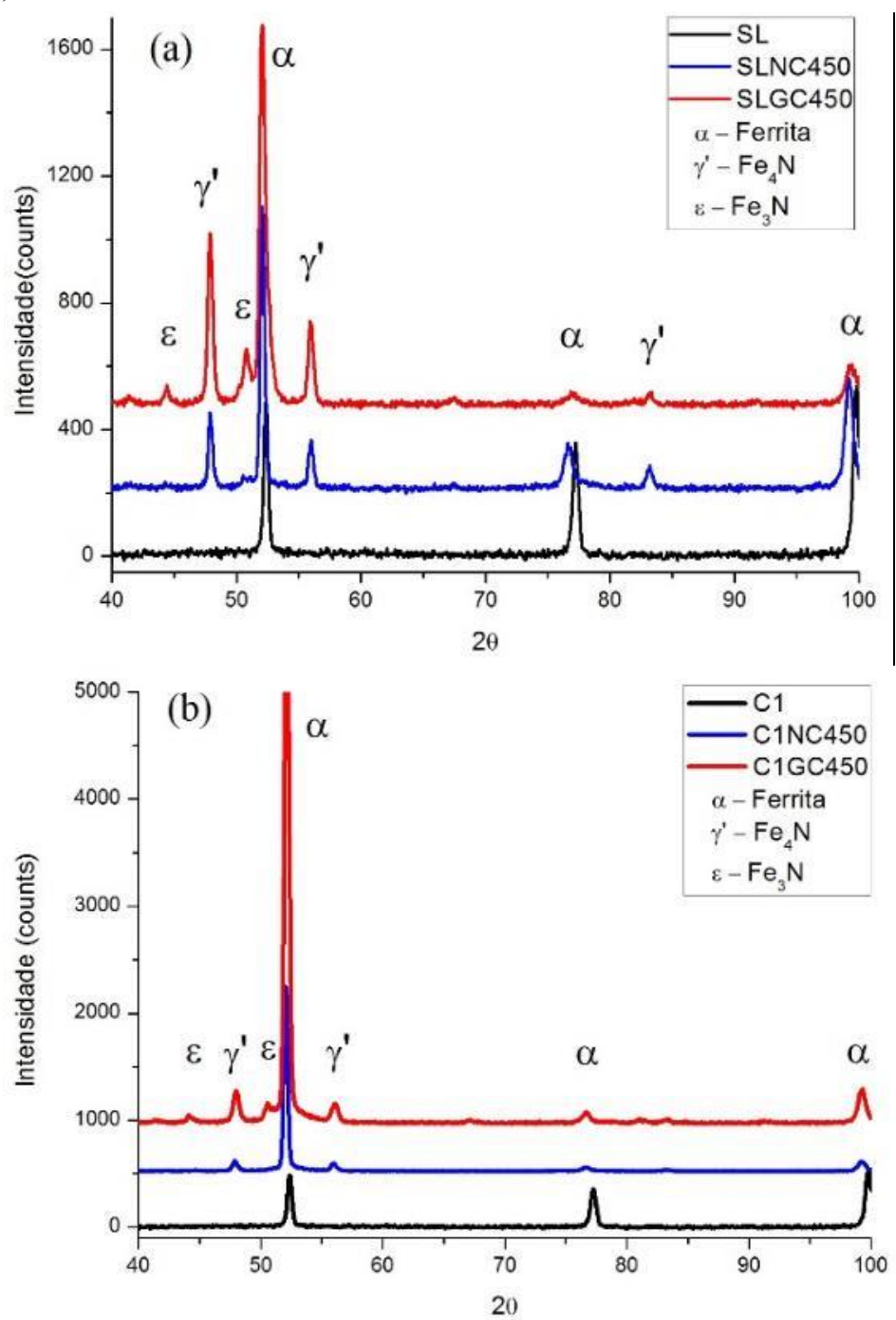

Figura 5: Padrões DRX das amostras antes e após a nitretação a plasma (a) convencional e (b) gaiola catódica. 


\section{DISCUSSÃO}

O material como recebido (SL), figura 3 (a), possui microestrutura ferrita-perlita bandeada, onde colônias alinhadas de perlita se apresentam entre camadas de ferrita. Na Figura 3 (b), que representa a condição de laminação $\mathrm{C} 1$, pode-se perceber a mesma microestrutura do material inicial, porém com reduzido bandeamento, as colônias de perlita aparecem discretamente mais dispersas se comparadas ao material inicial. Esta leve alteração microestrutural pode ser importante no aumento da resistência à fragilização por hidrogênio, pois a microestrutura exerce papel importante na influência ao TIH, uma vez que ela determinará a densidade e os tipos de sítios de aprisionamento de $\mathrm{H}$ [9].

A norma ASTM 6148-97 classifica os sítios de aprisionamento de hidrogênio como reversíveis e irreversíveis, levando em conta a energia de ligação do sítio com H e o tempo de permanência do átomo no "trap" $[30,31]$. As interfaces carbeto-ferrita em aços perlíticos são sítios preferenciais para o acúmulo irreversível de hidrogênio, e regiões de bandeamento apresentam maior quantidade desse tipo de interface, além do mais, microestruturas alinhadas (segregação), promovem caminhos de propagação de trincas [10,32-34]. Dessa forma, a maior distribuição da fase perlita causada pela laminação a morno da condição $\mathrm{C} 1$ sugere um aumento da resistência ao $\mathrm{TIH}$, porém se faz necessário realizar ensaio para confirmar o proposto.

A formação da camada de compostos observada nas imagens, Figuras 3 (c), (d), (e), e (f), é de grande importância no aumento da resistência ao TIH, pois é considerada a principal barreira contra a penetração do hidrogênio, os valores de espessura de camada de compostos estão de acordo com trabalhos realizados por outros autores [35, 36, 37]. De acordo com a Tabela 3, nota-se que tratamentos com gaiola catódica (SLGC450 e C1GC450), apresentaram camadas com uniformidade semelhante e com maior espessura se comparadas com os tratamentos a plasma convencionais (SLNC450 e C1NC450), essa maior espessura de camada de compostos nas amostras nitretadas com gaiola catódica também é um outro parâmetro importante no combate aos efeitos deletérios do hidrogênio, camada de compostos de maior espessura diminuem a permeabilidade de $\mathrm{H}$ [38]. A maior espessura pode ser explicada pela diferença no processo de formação da camada em cada tipo de tratamento. Na nitretação a plasma convencional, o sputtering atua diretamente no material nitretado, havendo dessa forma, uma concorrência entre a formação e dissociação da camada superficial, já no processo com gaiola catódica, o bombardeamento iônico ocorre na tela ativa (gaiola), não havendo dissociação da camada formada $[25,39,40]$.

A diferença entre a microdureza das amostras SL e C1 pode estar relacionada com a leve modificação microestrutural causada pela aplicação de laminação a morno, outras fases dispersas também podem estar presentes na matriz, como bainita superior e componentes de martensita-austenita, não detectáveis por microscopia ótica [41]. Ainda observando a Figura 4, podemos perceber que as amostras tratadas com gaiola catódica formaram superfícies com maior dureza que os tratamentos convencionais, esse fato está relacionado com a maior espessura de camada de compostos e as fases que a compõem, como será mostrado posteriormente.

Com exceção da amostra C1NC450, nota-se que quanto maior a espessura da camada de compostos, maior e a microdureza superficial. Segundo a norma ASTM E-384 e outros autores [42, 43], durante a aferição de microdureza de revestimentos superficiais se deve utilizar cargas baixas para que a penetração do indentador não ultrapasse uma profundidade máxima de 1/10 da espessura de camada. Dessa forma, ao confrontar os altos valores de microdureza obtidos na Fig. 4 com os baixos valores de espessura de compostos apresentados na Figura 3 e Tabela 3, podemos admitir que o indentador pode ter ultrapassado a camada de compostos, entrando na região do substrato, sugerindo a formação de zona de difusão [44]. Essa pode ser a explicação para o valor superior de microdureza da amostra C1NC450 mesmo possuindo a menor camada de compostos.

As fases $\mathrm{Fe}_{4} \mathrm{~N}\left(\gamma^{\prime}\right)$ e $\mathrm{Fe}_{2-3} \mathrm{~N}(\varepsilon)$, observadas na Fig. 5, pertencem à camada de compostos, já a ferrita $(\alpha)$, surge como contribuição do material do substrato sob a camada de compostos de pequena espessura no material nitretado, conforme mostra a Fig. 3. Outros autores [35-37] também observaram a formação dessas fases em camada de compostos que diminuíram a permeação por hidrogênio. Pode-se perceber um deslocamento dos picos referentes a fase ( $\alpha$ ) para ângulos menores, isso acontece devido a distorção causada pelos átomos de nitrogênio na estrutura cristalina do ferro, reforçando ainda mais a ideia de formação de zona de difusão no material nitretado.

Ao analisar os difratogramas das amostras submetidas a nitretação a plasma convencional (traço azul) nas Figuras 5 (a) e (b), percebe-se a que a camada de compostos é predominantemente formada por nitreto $\mathrm{Fe}_{4} \mathrm{~N}\left(\gamma^{\prime}\right)$. Diferentemente, as amostras nitretadas com gaiola catódica (traço vermelho), além da fase $\gamma^{\prime}$, está presente também a fase $\mathrm{Fe}_{3} \mathrm{~N}(\varepsilon)$ que possui maior dureza e resistência à corrosão que a primeira [45], o que também pode ter contribuído para a dureza superior nas amostras nitretadas com esse dispositivo. Esse fenô- 
meno ocorre porque no método convencional o sputtering causa descarbonetação superficial, o que favorece a formação de $\mathrm{Fe}_{4} \mathrm{~N}\left(\gamma^{\prime}\right)$. Já no processo com gaiola catódica, o bombardeamento atua na tela e não causa descarbonetação superficial, um maior conteúdo de carbono favorece a formação de camada bifásica $\mathrm{Fe}_{4} \mathrm{~N}$ $\left(\gamma^{\prime}\right)$ e $\mathrm{Fe}_{3} \mathrm{~N}(\varepsilon)[21]$.

\section{CONCLUSÕES}

A aplicação de laminação a morno provocou uma diminuição do bandeamento observado na microestrutura do material como recebido através de uma maior distribuição da fase perlita. A nitretação a plasma convencional e com gaiola catódica produziu um aumento máximo da microdureza superficial do aço em questão de até $350 \%$ através da formação de zona de difusão e camadas de compostos de $0,8 \mu \mathrm{m}$ espessura mínima e 3,3 $\mu \mathrm{m}$ de espessura máxima, sendo os maiores valores observados no material nitretado com gaiola catódica. A análise DRX revelou que as camadas de compostos eram formadas basicamente por nitretos $\mathrm{Fe}_{4} \mathrm{~N}\left(\gamma^{\prime}\right)$ e $\mathrm{Fe}_{3} \mathrm{~N}(\varepsilon)$. Todas essas características microestruturais e superficiais são conhecidas por aumentar a resistência à fragilização por hidrogênio, dessa forma, a combinação de laminação a morno e nitretação a plasma convencional e, principalmente com gaiola catódica, pode ser promissora no objetivo de diminuir os efeitos deletérios provocados pelo hidrogênio.

\section{AGRADECIMENTOS}

Agradecimento especial à CAPES por financiar parte desse trabalho.

\section{BIBLIOGRAFIA}

[1] FALLAHMOHAMMADI, E., BOLZONI, F., FUMAGALLI, G., et al., "Hydrogen diffusion into three metallurgical microstructures of a C-Mn X65 and low alloy F22 sour service steel pipelines", International Journal of Hydrogen Energy, v. 39, n. 25, pp. 13300-13313, 2014.

[2] ZHANG, T., ZHAO, W., LI, T., et al., "Comparison of hydrogen embrittlement susceptibility of three cathodic protected subsea pipeline steels from a point of view of hydrogen permeation", Corrosion Science, v. 131, pp. 104-115, 2018.

[3] MOHTADI-BONAB, M.A., SZPUNAR, J.A., BASU, R., et al., "The mechanism of failure by hydrogen induced cracking in an acidic environment for API 5L X70 pipeline steel", International journal of hydrogen energy, v. 40, n. 2, pp. 1096-1107, 2015.

[4] FIGUEREDO, R.M., DE OLIVEIRA, M.C., DE PAULA, L.J., et al., "A Comparative Study of Hydrogen-Induced Cracking Resistances of API 5L B and X52MS Carbon Steels”, International Journal of Corrosion, 2018.

[5] NATIVIDAD, C., GARCIA, R., LÓPEZ, V.H., et al., Mechanical and Metallurgical Properties of Grade X70 Steel Linepipe Produced by Non-conventional Heat Treatment. In: Characterization of Metals and Alloys. $1^{\circ}$ ed; Switzerland; Springer, Cham, pp. 3-11, 2017.

[6] DONG, C.F., LIU, Z.Y., LI, X.G. et al., "Effects of hydrogen-charging on the susceptibility of X100 pipeline steel to hydrogen-induced cracking", International journal of hydrogen energy, v. 34, n. 24, pp. 9879-9884, 2009.

[7 SERNA, S., MARTINEZ, H., LÓPEZ, S.Y., et al., "Electrochemical technique applied to evaluate the hydrogen permeability in microalloyed steels", International Journal of Hydrogen Energy, v. 30, n. 12, pp. 1333-1338, 2005.

[8] NACE International, "Evaluation of Pipe line and pressure vessel steel for resistance to hydrogen-induced crack,” Tech. Rep. ANSI/NACE TM0284, NACE International, Houston, Tex, USA, 2017.

[9] ZHAO, M.C., YANG, K., SHAN, Y.Y., et al., "Comparison on strength and toughness behaviors of microalloyed pipeline steels with acicular ferrite and ultrafine ferrite", Materials Letters, v. 57, n. 9-10, pp. 1496-1500, 2003.

[10] HINCAPIE-LADINO, D., FALLEIROS, N. A., "Trincamento induzido por hidrogênio em aços microligados”. Tecnologia em Metalurgia, Materiais e Mineração, v. 12, n. 1, 2015.

[11] PEREIRA, V. S. M., Efeito de características microestruturais na difusividade do hidrogênio em dois aços grau API X65. D. Sc., USP, São Paulo, 2017. 
[12] NAFISI, S., ARAFIN, M.A., COLLINS, L., et al., "Texture and mechanical properties of API X100 steel manufactured under various thermomechanical cycles”, Materials Science and Engineering, v. 531, pp. $2-11,2012$.

[13] MASOUMI, M., SILVA, C. C., ABREU, H. F. G., "Effect of crystallographic orientations on the hydrogen-induced cracking resistance improvement of API 5L X70 pipeline steel under various thermomechanical processing”, Corrosion Science, v. 111, pp. 121-131, 2016.

[14] FORCEY, K. S., ROSS, D. K., WU, C. H., "The formation of hydrogen permeation barriers on steels by aluminizing”, Journal of Nuclear Materials, v. 182, pp. 36-51, 1991.

[15] ZHANG, T., ZHAO, W., ZHAO, Y., et al., "Effects of surface oxide films on hydrogen permeation and susceptibility to embrittlement of X80 steel under hydrogen atmosphere", International Journal of Hydrogen Energy, v. 43, n. 6, pp. 3353-3365, 2018.

[16] YAMABE, J., MATSUOKA, S., MURAKAMI, Y., "Surface coating with a high resistance to hydrogen entry under high-pressure hydrogen-gas environment", International journal of hydrogen energy, v. 38, n. 24, pp. 10141-10154, 2013.

[17] MICHLER, T., "Influence of plasma nitriding on hydrogen environment embrittlement of 1.4301 austenitic stainless steel”, Surface and Coatings Technology, v. 202, n. 9, pp. 1688-1695, 2008.

[18] JONES, C.K., MARTIN, S.W., STURGES, D.J., et al: - Heat treatment'73. Anais de congresso, Ed. The Metals Society, pp. 71-77, Londres, dezembro de 1973.

[19] PREVEY, P.P., JAYARAMAN, N.J., ONTKO, N.O., et al., "Mechanical Suppression of SCC and Corrosion Fatigue Failures in 300M Steel Landing Gear". ASIP, n. 254, Tennessee, EUA, December of 2004.

[20] SIENZ, S., MÄNDL, S., RAUSCHENBACH, B., "In situ stress measurements during low-energy nitriding of stainless steel”, Surface and Coatings Technology, v. 156, n. 1-3, pp. 185-189, 2002.

[21] DE SOUSA, R.R.M., MOURA, Y.J.L., SOUSA, P.A., et al., "Nitriding of AISI 1020 steel: comparison between conventional nitriding and nitriding with cathodic cage", Materials Research, v. 17, n. 3, pp. 708713,2014

[22] LI, C. X., GEORGES, J., LI, X. Y., "Active screen plasma nitriding of austenitic stainless steel”, Surface Engineering, v. 18, n. 6, pp. 453-457, 2002.

[23] LI, C. X., BELL, T., "Corrosion properties of active screen plasma nitrided 316 austenitic stainless steel”, Corrosion Science, v. 46, n. 6, pp. 1527-1547, 2004.

[24] ALVES JR, C., SILVA, E. F., MARTINELLI, A. E., "Effect of workpiece geometry on the uniformity of nitrided layers", Surface and Coatings Technology, v. 139, n. 1, pp. 1-5, 2001.

[25] ALVES JR, C., DE ARAÚJO, F.O., RIBEIRO, K.J.B., et al., "Use of cathodic cage in plasma nitriding”, Surface and Coatings Technology, v. 201, n. 6, pp. 2450-2454, 2006.

[26] DE SOUSA, R.R.M., DE ARAÚJO, F.O., DA COSTA, J.A., et al., "Deposition of TiO2 Film on Duplex Stainless Steel Substrate Using the Cathodic Cage Plasma Technique", Materials Research, v. 19, n. 5, pp. 1207-1212, 2016.

[27] LOPES, R.F., DA COSTA, J.A., SILVA, W., et al., " $\mathrm{TiO}_{2}$ anti-corrosive thin films on duplex stainless steel grown using cathodic cage plasma deposition", Surface and Coatings Technology, v. 347, pp. 136-141, 2018.

[28] FARAHAT, A. I. Z. "Dilatometry determination of phase transformation temperatures during heating of $\mathrm{Nb}$ bearing low carbon steels", Journal of materials processing technology, v. 204, n. 1-3, pp. 365-369, 2008.

[29] DE SOUSA, R.R.M., MENDES, M., VALADÃO, E., et al., "Nitretação iônica em gaiola catódica do aço ferramenta tipo AISI D2 para trabalho a frio", Revista Matéria, v. 14, n. 2, p. 861-868, 2009.

[30] ASTM. G148-97. Standard Practice for Evaluation of Hydrogen Uptake, Permeation, and Transport in Metals by an Electrochemical Technique, ASTM International, West Conshohocken, PA, USA, 2003.

[31] THOMAS, R.L., LI, D., GANGLOFF, R.P., et al., "Trap-governed hydrogen diffusivity and uptake capacity in ultrahigh-strength AERMET 100 steel”, Metallurgical and Materials Transactions A, v. 33, n. 7, p. 1991-2004, 2002.

[32] WANG, S.H., LUU, W.C., HO, .F., et al., "Hydrogen permeation in a submerged arc weldment of TMCP steel", Materials Chemistry and Physics, v. 77, n. 2, pp. 447-454, 2003.

[33] LIOU, H. Y., WANG, S. C., HSIEH, R. I., "Effects of microstructures on hydrogen embrittlement and absorbed energies in a low carbon steel”, China Steel Technical Report(Taiwan), v. 10, pp. 22-31, 1996. 
[34] ELBOUJDAINI, M.; REVIE, R. W., "Metallurgical factors in stress corrosion cracking (SCC) and hydrogen-induced cracking (HIC)", Journal of solid state electrochemistry, v. 13, n. 7, pp. 1091-1099, 2009.

[35] SILVA JUNIOR, A. J. D., Influência de nitretação à plasma sobre a resistência à fadiga do aço API 5L grau B., Tese de D.Sc., UNB, Brasília, 2016.

[36] ZAMPRONIO, M. A., FASSINI, F. D., MIRANDA, P. E. V., "Design of ion-implanted hydrogen contamination barrier layers for steel", Surface and Coatings Technology, v. 70, n. 2-3, pp. 203-209, 1995.

[37] BOTT, A.H., BRÜHL, S.P., GÓMEZ, B., et al., "Pulsed-plasma-nitrided API 5L X-65 steel: hydrogen permeability and microstructural aspects", Journal of Physics D: Applied Physics, v. 31, n. 24, pp. 3469, 1998.

[38] BRUZZONI, P., BRÜHL, S.P., GÓMEZ, P., et al., "Hydrogen permeation modification of 4140 steel by ion nitriding with pulsed plasmas", Surface and Coatings Technology, v. 110, n. 1-2, pp. 13-18, 1998.

[39] DE SOUSA, R.R.M., DE ARAÚJO, F.O., DA COSTA, J.A., et al., "Cathodic Cage plasma nitriding: An innovative technique", Journal of Metallurgy, v. 2012, n. 385963, 2012.

[40] MÖLlER, W., PARASCANDOLA, S., TELBIZOVA, T., et al., "Surface processes and diffusion mechanisms of ion nitriding of stainless steel and aluminium", Surface and Coatings Technology, v. 136, n. 1-3, pp. 73-79, 2001.

[41] MASOUMI, M., HERCULANO, L.F., ALMEIDA, A.A., et al., "Texture Study Across Thickness of API X70 Steel After Hot Deformation and Different Posttreatments", JOM, v. 68, n. 1, pp. 401-409, 2016.

[42] JOHNSON, K. L., "The correlation of indentation experiments", Journal of the Mechanics and Physics of Solids, v. 18, n. 2, pp. 115-126, 1970.

[43] ZIELINSKI, W., HUANG, H., GERBERICH, W. W., "Microscopy and microindentation mechanics of single crystal $\mathrm{Fe}^{-} 3$ wt.\% Si: Part II. TEM of the indentation plastic zone", Journal of materials research, v. 8, n. 6, pp. 1300-1310, 1993.

[44] ALLENSTEIN, A.N., LEPIENSKI, C.M., BUSCHINELLI, A.J., et al., "Plasma nitriding of CA-6NM steel: effect of $\mathrm{H} 2+\mathrm{N} 2$ gas mixtures in nitride layer formation for low $\mathrm{N} 2$ contents at $500{ }^{\circ} \mathrm{C} "$, Materials Research, v. 13, n. 4, p. 557-562, 2010.

[45] VERMA, R. Plasma nitriding : State -of - the - art Industrial Heating, pp.8-14, Sept. 1985.

\section{ORCID}

André Sales Aguiar Furtado João Rodrigues de Barros Neto Petteson Linniker Carvalho Serra Rômulo Ribeiro Magalhães de Sousa https://orcid.org/0000-0001-6848-3406

https://orcid.org/0000-0003-2692-0211

https://orcid.org/0000-0002-0030-3496

https://orcid.org/0000-0003-2062-6505 